\title{
Influence of the porosity on diffusion and lifetime in porous $\mathrm{TiO}_{2}$ layers
}

\author{
Th. Dittrich ${ }^{\mathrm{a}), \mathrm{b})}$ \\ Hahn-Meitner-Institute, Glienicker Strasse 100, 14109 Berlin, Germany
}

A. Ofir, S. Tirosh, L. Grinis, and A. Zaban ${ }^{\mathrm{a}), \mathrm{c})}$

Chemistry Department, Bar Ilan University, 52900 Ramat Gan, Israel

(Received 4 January 2006; accepted 5 April 2006; published online 5 May 2006)

\begin{abstract}
Photocurrent transients were investigated on pressed and sintered porous $\mathrm{TiO}_{2}$ layers which were immersed in electrolyte during the measurements. Unpressed porous $\mathrm{TiO}_{2}$ layers were prepared by electrophoretic deposition. The porosity of the layers was changed systematically by pressing. The surfaces of the $\mathrm{TiO}_{2}$ nanoparticles and the intimate contact between them were identically conditioned by sintering in air at $450{ }^{\circ} \mathrm{C}$ after pressing. With decreasing porosity, the diffusion coefficient increased while the electron lifetime decreased. The results are discussed on the base of the change of the mean coordination number between $\mathrm{TiO}_{2}$ nanoparticles. (C) 2006 American Institute of Physics. [DOI: 10.1063/1.2201045]
\end{abstract}

The transport behavior of excess charge carriers in porous semiconductors is of great practical and fundamental interest, for various applications such as sensors ${ }^{1}$ or dyesensitized solar cells. ${ }^{2}$ In porous silicon, ${ }^{3}$ for example, the photoluminescence increases with decreasing conductivity ${ }^{4}$ since excess electrons and holes recombine radiatively with each other in the same spatial unit. The situation is different for porous $\mathrm{TiO}_{2}$ layers immersed in electrolyte. In such case, excess electrons and holes are trapped very rapidly at different sites and screened from each other with respect to the Debye screening length. Further, holes are lost into the electrolyte while electrons can diffuse through a porous $\mathrm{TiO}_{2}$ layer. Porous $\mathrm{TiO}_{2}$, in comparison with porous $\mathrm{Si}$, consists of a porous network of sintered nanoparticles. Thus, the porosity can be changed without changing the size of the interconnected nanoparticles in porous $\mathrm{TiO}_{2}$ layers. With respect to a random packing model, the mean coordination number between nanoparticles is given by the porosity. ${ }^{5}$ The porosity is strongly correlated with the diffusion coefficient due to percolation. ${ }^{6}$ The critical porosity, above which electrical transport becomes impossible, amounts to $P_{C}=0.76$ for porous $\mathrm{TiO}_{2}$ layers. ${ }^{6}$

In this work, porous $\mathrm{TiO}_{2}$ layers immersed in electrolyte are used as a model system to study the influence of the morphology of porous semiconductors on the diffusion of excess charge carriers. Diffusion coefficients are measured by time resolved photoconductivity.

Recently, electrophoretic deposition (EPD) was applied to produce porous $\mathrm{TiO}_{2}$ layers without using an organic binder. ${ }^{7}$ After EPD, the $\mathrm{TiO}_{2}$ nanoparticles are not yet well sintered to each other. Therefore, the porosity of the porous $\mathrm{TiO}_{2}$ layers can be systematically changed by pressing. In our experiments, porous $\mathrm{TiO}_{2}$ layers with an initial thickness of $L=9.7 \mu \mathrm{m}$ were densified to thicknesses of $8,6.9,6.4$, and $6.1 \mu \mathrm{m}$ by pressing with $0.2,0.4,0.6$, and 0.8 tons $/ \mathrm{cm}^{2}$ (the pressure is given in tons per $\mathrm{cm}^{2}$ ). The thicknesses were measured with a step profiler. The porosity of the unpressed porous $\mathrm{TiO}_{2}$ layer was $P=0.6$. Therefore, the porosities

\footnotetext{
a) Authors to whom correspondence should be addressed.

b)Electronic mail: dittrich@hmi.de

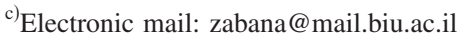

amounted to $0.51,0.44,0.39$, and 0.36 for the porous $\mathrm{TiO}_{2}$ layers pressed with $0.2,0.4,0.6$, and 0.8 tons $/ \mathrm{cm}^{2}$. The porosities of $0.6,0.51,0.44,0.39$, and 0.36 correspond to the mean coordination numbers $(K)$ of $4,4.9,5.9,6.8$, and 7.4 , in accordance to Ref. 5. Both the pressed and unpressed layers were identically sintered in air at $450{ }^{\circ} \mathrm{C}$ for $30 \mathrm{~min}$. The porous $\mathrm{TiO}_{2}$ layers with different porosities, were prepared on conductive electrodes (glass coated with $\mathrm{SnO}_{2}: \mathrm{F}$, TEC15). The transient photocurrent (PC) measurements were performed in a two electrode cell (Pt wire as counter electrode) with a quartz window. A $0.5 M \mathrm{NaCl}, p \mathrm{H}=2.0$ $(\mathrm{HCl})$ solution was used as conductive electrolyte. The porous $\mathrm{TiO}_{2}$ layers were sealed with an $\mathrm{O}$ ring (diameter of $7 \mathrm{~mm}$ ) and illuminated from the electrolyte side with pulses of a $\mathrm{N}_{2}$ laser (wavelength of $337 \mathrm{~nm}$, pulse duration of $5 \mathrm{~ns}$, repetition rate of $1 \mathrm{~Hz}$, and intensity of $40 \mu \mathrm{J} / \mathrm{cm}^{2}$ ). The photocurrent transients were recorded with a $100 \mathrm{MHz}$ personal computer card (gage) via a $50 \Omega$ resistance.

Figure 1 shows photocurrent transients of the unpressed porous $\mathrm{TiO}_{2}$ layer and of the pressed porous $\mathrm{TiO}_{2}$ layers with porosities of $0.51,0.44,0.39$, and 0.36 . In the following, only the part between $10^{-4}$ and $10^{-1} \mathrm{~s}$, which is related to electron diffusion, will be considered (the so-called diffusion

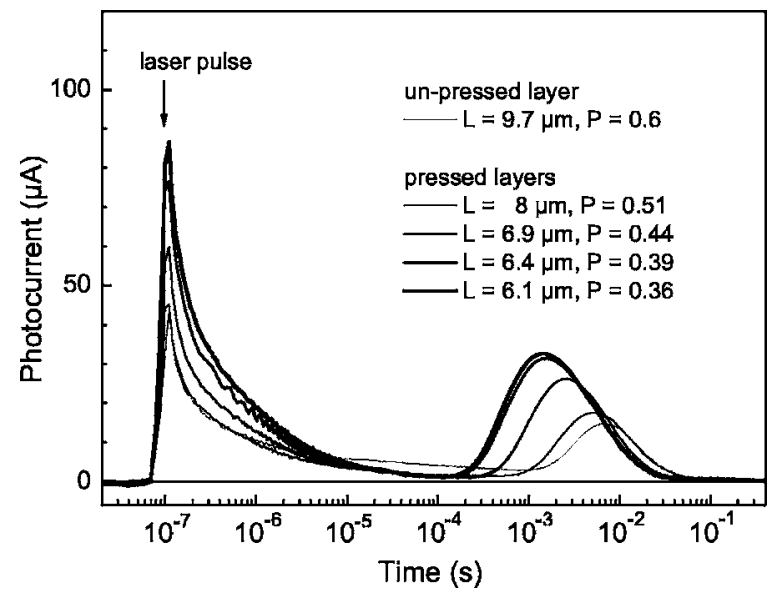

FIG. 1. Photocurrent transients of the unpressed porous $\mathrm{TiO}_{2}$ layer (porosity 0.6) and of the pressed porous $\mathrm{TiO}_{2}$ layers with porosities of $0.51,0.44$, 0.39 , and 0.36 . The arrow marks the onset of the laser pulse. 


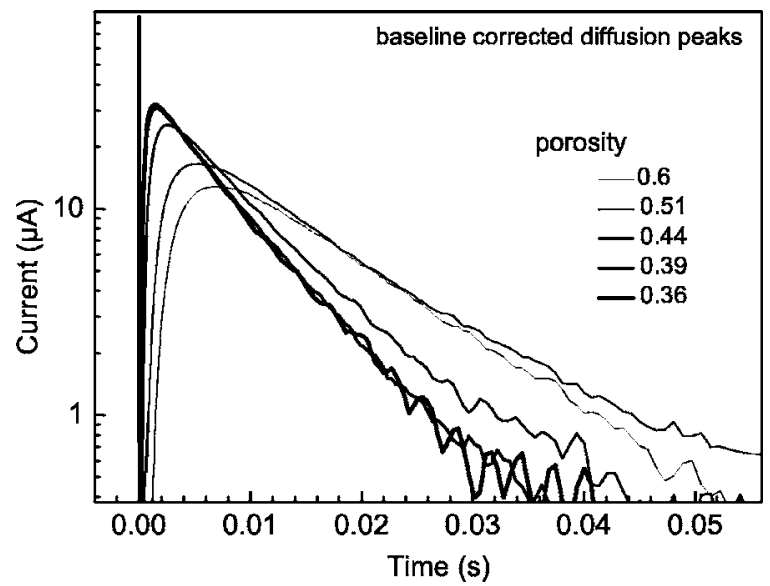

FIG. 2. Measured photocurrent transients (see Fig. 1) corrected to the base line in a log-linear scale.

peak). The increasing parts of the transients shift to shorter times with decreasing porosity due to faster diffusion. The faster diffusion is induced by the reduced thicknesses after pressing and by the increased diffusion coefficients. The decreasing parts of the diffusion peaks of the layers with $P=0.44,0.39$, and 0.36 shift slightly to shorter times in comparison with the transients of the porous $\mathrm{TiO}_{2}$ layers with $P=0.6$ and 0.51 . This shift is related to a decrease of the electron lifetime.

Figure 2 depicts the measured PC transients corrected to the base line in a log-linear scale. At longer times, a decay time constant can be obtained. This time constant does not show a real dependence on the porosity of the porous $\mathrm{TiO}_{2}$ layers. There are roughly two values of $\tau$ : (i) about $12 \mathrm{~ms}$ for the unpressed and pressed with moderate pressures porous $\mathrm{TiO}_{2}$ layers and (ii) about $7 \mathrm{~ms}$ for porous $\mathrm{TiO}_{2}$ layers pressed with pressures higher than 0.2 tons $/ \mathrm{cm}^{2}$. Since there is no correlation with the porosity, the change of the decay time constant has to be related to a qualitative change of the defect structure in $\mathrm{TiO}_{2}$ particles due to local deformations during processing. The defects are not healed during the sinter process.

Figure 3(a) gives the porosity dependence of the accumulated charge (integrated over the diffusion peak of the PC transients). The accumulated charge was of the order of $0.2 \mu \mathrm{A}$ s. It increased for the pressed electrodes by about $25 \%$ and decreased slightly with decreasing porosity.

For obtaining an effective diffusion coefficient $\left(D_{\text {eff }}\right)$, the time position of the diffusion peak is usually analyzed, ${ }^{8}$

$$
D_{\text {eff }}=\frac{L^{2}}{6 t_{\text {peak }}} .
$$

It has been also proposed to analyze the exponential decay at the long times of the $\mathrm{PC}$ transients for extracting the effective diffusion coefficient, ${ }^{9}$

$$
D_{\mathrm{eff}}^{*} \cong \frac{L^{2}}{2.35 \tau} .
$$

The porosity dependencies of $L^{2} /(2.35 \tau)$ and $D_{\text {eff }}$ are shown in Figs. 3(b) and 3(c). It could not be observed a pronounced dependence of $L^{2} /(2.35 \tau)$ or the porosity in our experiments. This means that the decaying part of the $\mathrm{PC}$ Downloaded 30 Aug 2007 to 134.30.24.17. Redistribution subject

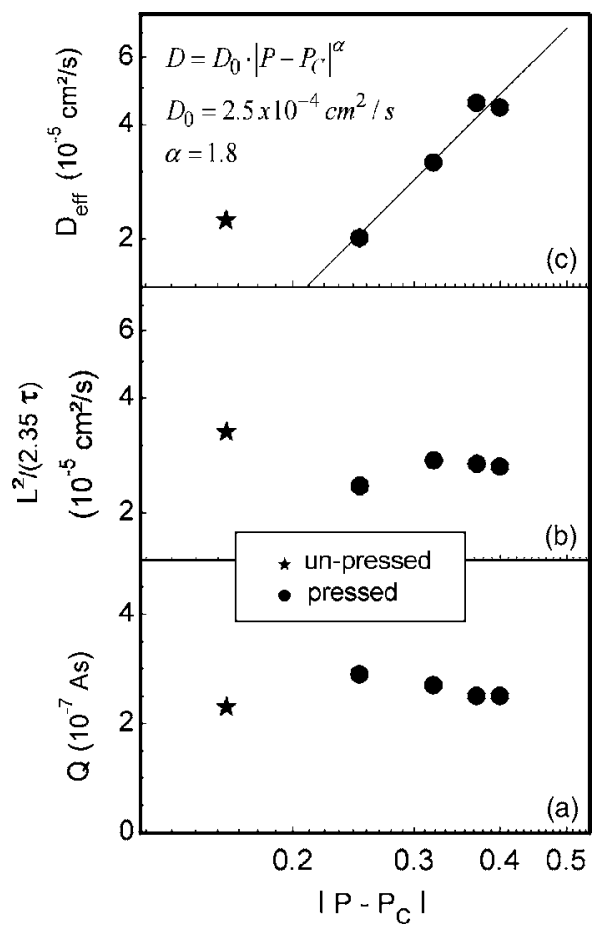

FIG. 3. Plot of the accumulated charge of the diffusion peak (a), of the squared layer thickness divided by the decay time constant (b), and of the effective diffusion coefficient (c) as a function of the porosity. The critical porosity was set to 0.76 (Ref. 6). The straight line in (c) gives the fit according to Eq. (4).

transients seems to be more controlled by modified trapping than by the diffusion process under the given conditions as mentioned above.

$D_{\text {eff }}$ increases with decreasing porosity for the pressed samples. $D_{\text {eff }}$ amounts to about $2 \times 10^{-5} \mathrm{~cm}^{2} / \mathrm{s}$ for the highest and $4.5 \times 10^{-5} \mathrm{~cm}^{2} / \mathrm{s}$ for the lowest porosities. As remark, the increasing part of the PC transients can be analyzed as well by using the approximation, ${ }^{8}$

$$
i(L, t)=\frac{q N_{0} L}{2 \cdot \sqrt{\pi D t}} \exp \left(-\frac{L^{2}}{4 D t}\right),
$$

where $q$ is the elementary charge and $N_{0}$ is the initial electron concentration. In such a case, a maximal diffusion coefficient can be defined. The values of the maximal diffusion coefficients amount, for example, to $2.5 \times 10^{-5}$ and 8 $\times 10^{-5} \mathrm{~cm}^{2} / \mathrm{s}$ for the samples with the highest and lowest porosities. The difference between $D_{\text {eff }}$ and the maximal diffusion coefficient is caused by dispersive transport.

For the pressed porous $\mathrm{TiO}_{2}$ layers, the dependence of $D_{\text {eff }}$ on the porosity can be described by a power law as supposed by Benkstein et al., ${ }^{6}$

$$
D_{\text {eff }}=D_{0}\left|P-P_{C}\right|^{\alpha} \text {. }
$$

The values of the parameters $D_{0}$ and $\alpha$ are 2.5 $\times 10^{-4} \mathrm{~cm}^{2} / \mathrm{s}$ and 1.8. If analyzing the maximal diffusion coefficient instead of $D_{\text {eff }}$, the values of $\alpha$ and $D_{0}$ amount to 2.2 and $8 \times 10^{-4} \mathrm{~cm}^{2} / \mathrm{s}$. The values of $\alpha$ and $D_{0}$ will depend sensitively on the value of $P_{C}$. Depending on the applied structure, the value of $P_{C}$ can change, for example, between 0.69 and $0.75 .{ }^{10}$ For comparison, $\alpha$ was 0.82 for screen printed porous $\mathrm{TiO}_{2}$ layers. ${ }^{6}$ A value of $\alpha$ of the order of 2 seems reasonable from percolation theory. ${ }^{10}$ There is a principal difference between pressed and unpressed porous $\mathrm{TiO}_{2}$
AlP license or copyright, see http://apl.aip.org/apl/copyright.jsp 
layers since the diffusion coefficient obtained for the unpressed layer does not follow the common dependence described in Eq. (4) for the pressed layers. This seems not surprising because pressing may strongly influence $D_{0}$ in general due to cracking of the initial structure. A question is whether pressing might change or not the percolation threshold due to introduction of a certain anisotropy.

The coordination number between $\mathrm{TiO}_{2}$ nanoparticles has been changed systematically by pressing porous $\mathrm{TiO}_{2}$ layers as mentioned above. We found a correlation between the porosity, i.e., the mean coordination number, and the diffusion coefficient for porous $\mathrm{TiO}_{2}$ layers which were densified by pressing. The results are of interest, for example, for the further development of dye-sensitized solar cells in which nanoporous $\mathrm{TiO}_{2}$ layers are used.

The authors are grateful to S. Dube and S. Bönisch from the Hahn-Meitner-Institute for implementing a system for logarithmic transient measurements. One of the authors
(A.Z.) acknowledges the financial support provided by the Israel Science Foundation founded by The Israel Academy of Science and Humanities.

${ }^{1}$ See, for example, W. Göpel and G. Reinhardt, in Sensors Update, edited by H. Baltes, W. Göpel, and J. Hesse (VCH, Weinheim, 1996).

${ }^{2}$ B. O'Regan and M. Grätzel, Nature (London) 353, 737 (1991).

${ }^{3}$ See, for example, A. G. Cullis, L. T. Canham, and P. D. J. Calcott, J. Appl. Phys. 82, 909 (1997).

${ }^{4}$ M. Ben-Chorin, A. Kux, and I. Schechter, Appl. Phys. Lett. 64, 481 (1993).

${ }^{5}$ J. van de Lagemaat, K. D. Benkstein, and A. J. Frank, J. Phys. Chem. B 105, 12433 (2001).

${ }^{6}$ K. D. Benkstein, J. Kopidakis, J. van de Lagemaat, and A. J. Frank, J. Phys. Chem. B 107, 7759 (2003).

${ }^{7}$ L. Grinis, A. Ofir, and A. Zaban (unpublished); J.-H. Yum, S.-S. Kim, D.-Y. Kim, and Y.-E. Sung, J. Photochem. Photobiol., A 173, 1 (2005).

${ }^{8}$ A. Solbrand, H. Lindström, H. Rensmo, A. Hagfeldt, and S.-E. Lindquist, J. Phys. Chem. B 101, 2514 (1997).

${ }^{9}$ J. van de Lagemaat and A. J. Frank, J. Phys. Chem. B 105, 11194 (2001).

${ }^{10}$ D. Stauffer and A. Aharony, Introduction to Percolation Theory, 2nd ed. (Taylor \& Francis, London, 1992). 